\title{
LA CALIDAD DE LOS SERVICIOS DE LAS EMPRESAS DE TURISMO ACTIVO EN PORTUGAL
}

\author{
Pedro Bento* \\ Instituto Politécnico de Beja. Portugal \\ https://orcid.org/0000-0002-3826-4357 \\ Luis Murta* \\ Instituto Politécnico de Beja. Portugal \\ https://orcid.org/0000-0002-4395-2664 \\ Jesús Sáez-Padilla** \\ Universidad de Huelva \\ https://orcid.org/0000-0002-7717-2121
}

\section{RESUMEN}

El turismo de naturaleza es uno de los sectores del turismo que más crece en el mundo. Este tipo de práctica aglutina muchas modalidades y actividades. Por ello, es importante investigar la forma como las empresas expertas en el área del turismo proporcionan los servicios, se identificaron los parámetros y condicionantes que determinan la calidad, proponiendo mejoras en los procedimientos. Se aplicó el cuestionario HEVA (Bento et al., 2017), adaptado de Mediavilla (2010), a 183 empresas de turismo activo portuguesas. Se ha descrito el perfil y opinión de los responsables de las empresas, destacando: el crecimiento del sector en Portugal, la importancia atribuida a instrumentos fiables, capaces de medir la satisfacción de los clientes, el compartir información inherente a la seguridad de los servicios y poseer recursos humanos especializados y motivados, son factores esenciales a la calidad de los servicios prestados por las empresas en el área.

Palabras clave: Deportes; naturaleza; turismo; calidad.

Fecha de recepción: 22 de mayo de 2018

Fecha de aceptación: 20 de diciembre de 2018

* Instituto Politécnico de Beja. Portugal. E-mail: pbento@ipbeja.pt, 1murta@ipbeja.pt

** Universidad de Huelva. Campus El Carmen. Avda. Tres de marzo, s/n. HUELVA (España). E-mail: jesus. saez@dempc.uhu.es 
ABSTRACT

\section{Service quality in Portuguese sport tourism companies}

Nature Tourism is one of the fastest growing sectors in the tourism area. These activities are carried out in a very particular environment. In this context, it is important to study the way in which expert companies in active tourism area provide their services, they were identified the parameters and constraints which determine the quality of the services they render, and subsequently propose improvements in processes and procedures. In this study, we analyze the portuguese active tourism companies profile $(\mathrm{N}=183)$, with the application of the validated HEVA questionnaire (Bento et al., 2017), from the original Mediavilla (2010). Among other results, the study showed the recent growth of this sector in Portugal, the importance of measuring customer satisfaction, sharing security activities information with clients and motivated human resources are essential factors in the quality of services provided by companies in the area.

Keywords: Sports; nature; tourism; quality.

\section{INTRODUCCIÓN}

De la asociación entre el turismo y el deporte, Alves (2010) defiende que surgieron comportamientos diferentes, donde la demanda por los espacios naturales y el descubrimiento de nuevos territorios, hábitos, culturas y costumbres aumentó, existiendo hoy el desarrollo masivo de servicios turísticos, capaces de caracterizar diferentes destinos (Bell et al., 2007). El presente artículo se refiere a un sector del turismo reciente, denominado en varios países de Europa, como Turismo Activo (Beedie, Hudson, 2003; Arcos, 2004; Cunha, 2006; Carvalhinho, 2011; Mediavilla et al., 2014). En Portugal, este sector ha crecido en los últimos años, donde estudios apuntaban a la importancia del descubrimiento de lugares singulares, a través de actividades de turismo y deportes, destacando el carácter muy lúdico de este tipo de experiencia (García, 2001).

Asociado a este crecimiento, comenzaron a surgir algunos problemas, resultantes de la falta de regulación que existió, principalmente en el principio del siglo veintiuno. El acceso a este tipo de actividad y la creación de empresas en el sector, no iba acompañado de grandes estructuras económicas y físicas, además el nivel de formación específico era bajo y los apoyos administrativos y gubernamentales eran escasos.

Otro de los factores resultantes de este escenario ya explicado anteriormente, fueron los accidentes graves, con pérdidas humanas irreparables que ocurrieron en ese contexto, en varios países de Europa (Mediavilla et al., 2014). Por lo tanto, es urgente estudiar e investigar este sector específico del turismo, intentando percibir qué tipo de servicios y actividades estarían asociados a las empresas turísticas que operan en el sector.

En este contexto, y con la intención de evitar consecuencias y situaciones dramáticas en el servicio del turismo activo, es necesario una capacitación y calificación por parte de los responsables, así como un compromiso físico y psicológico por parte del cliente final. 
Todos estos factores suscitan la urgencia que había en estudiar e investigar mejor el sector, intentando identificar los parámetros y condicionantes que determinan la calidad de los servicios demandados por las empresas en el área.

En los últimos años, los trabajos realizados por Mediavilla (2010) culminaron con la concepción y validación de una herramienta capaz de medir los parámetros de calidad emergentes de los servicios de las empresas de turismo activo.

El principal objetivo de la investigación fue determinar la calidad de las empresas de turismo activo de Portugal utilizando una herramienta validada como el cuestionario HEVA.

A continuación, presentamos la herramienta HEVA adaptada a la lengua portuguesa, así como los resultados alcanzados, a través de las respuestas al cuestionario, donde participaron 183 empresas en Portugal.

\section{CUESTIONARIO HEVA}

El cuestionario HEVA (Bento et al., 2017), permite obtener los datos de las empresas de turismo activo, para posterior análisis de la calidad de las mismas. Su estructura y contenidos fueron adaptados y validados para la lengua portuguesa. Se elaboró la traducción y adaptación de los elementos presentes en cada parámetro teniendo en cuenta los destinatarios, así como el tratamiento y la validez formal de preguntas (Heinemann, 2008).

El cuestionario está dividido en 3 apartados. En el primer apartado, encontramos informaciones básicas acerca del perfil general de las empresas (dónde está registrada, número de socios, actividades disponibles, etc.).

En el segundo apartado de preguntas están presentes 7 parámetros multidimensionales, con 10 ítems cada uno, los cuales se pueden responder en una escala de Likert de 5 puntos, desde el 1 (poco importante) a 5 (muy importante), y otra escala de Gutman (Sí o no), que se responde de acuerdo con la implementación, o no, de cada ítem en la gestión de la empresa, por ejemplo: "Hacer un estudio de viabilidad empresarial".

Los parámetros multidimensionales son: Entidad, Actividad “Top”, Recursos Materiales, Seguridad, Medio Ambiente Natural, Recursos Humanos y Cliente. En este bloque, la puntuación de las respuestas puede ir de 70 a 350 puntos, teniendo en cuenta todos los elementos de los 7 parámetros multidimensionales.

En el tercer y último apartado, están presentes preguntas complementarias redactadas para conocer la opinión del responsable de la empresa, en relación a los aspectos que cree que sus clientes consideran más importantes. Se divide en 15 ítems (Amabilidad, Atención al Cliente, Interés de las Actividades, Capacidad de Respuesta, Conservación del Entorno, Eficacia en el Servicio, Instalaciones, Limpieza, Percepción de Garantías del Servicio, Productos Complementarios, Profesionalidad, Puntualidad, Satisfacción del producto, Seguridad y Tratamiento de las Reclamaciones), insertados en la misma escala de Likert explicada anteriormente. La puntuación máxima que se puede obtener es de 75 puntos y mínima de 15 puntos.

El análisis de los siete parámetros multidimensionales, presentes en el cuestionario HEVA (Bento et al., 2017), se tornó un buen método para investigar la valoración y percepción de la calidad del servicio, como un modelo multifuncional en el turismo activo portugués. 


\section{CALIDAD DE LOS SERVICIOS DE EMPRESAS DE TURISMO ACTIVO EN PORTUGAL: APLICACIÓN DE HEVA (BENTO ET AL., 2017) A 183 EMPRE- SAS DE TURISMO ACTIVO EN PORTUGAL}

En este estudio participaron 183 empresas de turismo activo, registradas en el Turismo de Portugal IP, través de la plataforma RNAAT (Registro Nacional de Empresas de Turismo Activo) (Portugal Turismo, 2015). Contestaron el cuestionario, empresas de 18 provincias de Portugal. La provincia de Faro, con 35 empresas $(19,1 \%)$, seguido de Lisboa con $24(13,1 \%)$ y Porto con $23(12,6 \%)$ fueron las regiones más representadas en este estudio.

\subsection{Procedimiento y análisis de los datos}

Para realizar la recogida de los datos, se pasó el cuestionario a las empresas, a través del envío en formato online, por la plataforma "Google Drive". Fue preocupación del equipo de investigación, la gestión del tiempo y la consecuente solicitud hecha a los responsables de las empresas, para contestar el cuestionario. De esta forma, se contactó las entidades, enviando el documento online, durante el período del año donde existe menor volumen de trabajo, especialmente entre noviembre y marzo (época de invierno). Enviamos y recogemos información, en un espacio temporal de 4 meses.

Para el análisis de los datos resultantes de las respuestas dadas al cuestionario HEVA, se recurrió a la literatura especializada (Fonseca, 2008, Maroco, 2011), se utilizaron las técnicas que más se ajustan a nuestro estudio y en función de los objetivos de nuestra investigación. Para el análisis descriptivo, a fin de caracterizar los datos obtenidos, desarrollamos tablas de frecuencia, representando porcentajes correspondientes y frecuencias absolutas. Se evaluaron los parámetros estadísticos de tendencia central y de dispersión, usamos diferentes tipos de gráficos, asegurando la representación más clara de los datos, para una mejor interpretación.

\section{RESULTADOS Y DISCUSIÓN}

Los primeros ítems del cuestionario permitieron caracterizar mejor el perfil de nuestra muestra, especialmente en lo que se refiere a la comunidad de registro de la empresa, su ámbito de intervención, el período de trabajo al año, productos ofertados, las actividades más demandadas, número de trabajadores, bases de operaciones, volumen económico y el tipo de intervención, de acuerdo con la opinión de sus responsables, que las mismas tienen en el área del turismo y/o deporte.

Presentamos también un análisis realizado a los ítems más contestados en cada uno de los parámetros, lo que permite conocer la opinión de los empresarios de turismo activo portugueses, participantes en el estudio con respecto a los aspectos de calidad, que ven como más importantes en sus servicios o productos. 


\subsection{Comunidad de registro}

Observando la figura 1, podemos ver la dispersión de la muestra, por las diversas comunidades de Portugal. Faro, con 35 empresas (19,1\%), seguido de Lisboa con 24 $(13,1 \%)$ y Porto con $23(12,6 \%)$ fueron las comunidades más representadas en este estudio.

\section{Figura 1}

COMUNIDAD DE REGISTRO DE LAS EMPRESAS PARTICIPANTES (N=183)

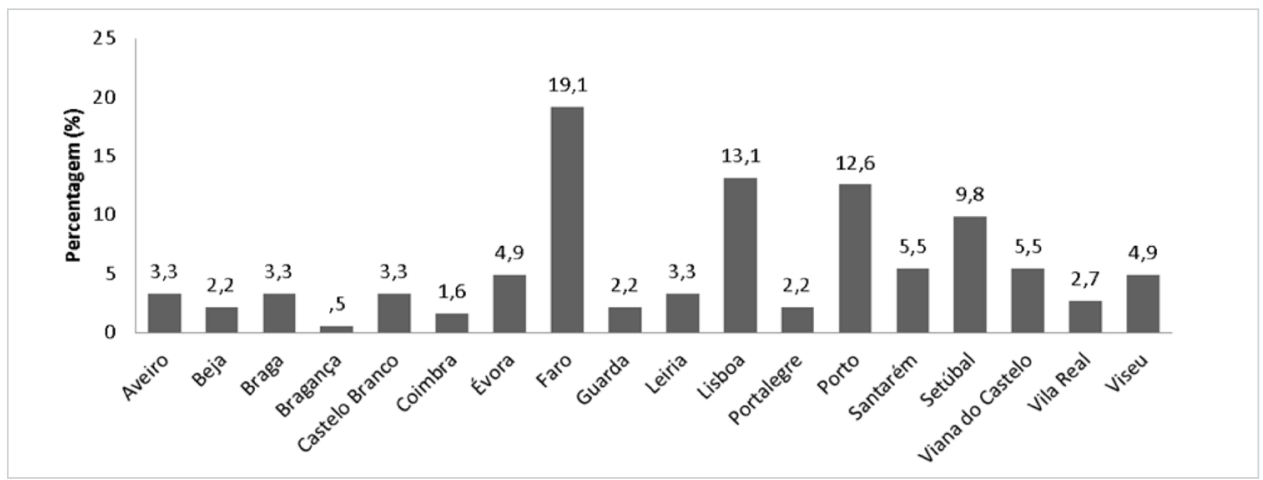

\section{2. Ámbito laboral y período de trabajo al año}

En este ítem se caracterizó el formato de intervención en el terreno de las empresas respondientes, la forma como dinamizan sus actividades / servicios en el territorio.

Tabla 1

ÁMBITO LABORAL

\begin{tabular}{lcc}
\hline Ámbito & Frecuencia & Porcentaje \\
\hline Local & 39 & $21,3 \%$ \\
\hline Provincial & 13 & $7,1 \%$ \\
\hline Autonómico & 50 & $27,3 \%$ \\
\hline Nacional & 47 & $25,7 \%$ \\
\hline Internacional & 34 & $18,6 \%$ \\
\hline Total & 183 & $100 \%$ \\
\hline
\end{tabular}

La mayoría de las empresas optaron por actuar en un ámbito más cercano, en relación con el local de su sede, gestionando y organizando actividades y servicios sin realizar grandes desplazamientos. Sin embargo, existen también un número importante de empresas que tienden a desplazarse de norte a sur del país $(25,7 \%)$, dinamizando 
sus actividades y servicios en un ámbito más nacional (tabla 1). También destacamos la internacionalización de 34 empresas, demostrando la importancia y valor del trabajo desarrollado por los responsables en el área. En cuanto a los resultados relativos al período de trabajo desarrollado a lo largo del año, verificamos que la mayoría de las empresas de turismo activo $(n=144)$, desarrollan su actividad durante los 12 meses del año (78,7\%). Sólo el $12 \%$ trabaja 6 meses al año y 5 empresas $(2,7 \%)$, afirman que desarrollan su actividad durante apenas 4 meses.

\subsection{Productos/actividades más demandadas y número de bases de operación}

El producto/actividad con más éxito o demanda, en las empresas participantes queda reflejado en la figura 2. Existen 15 ítems diferentes, lo que demuestra diferenciación de soluciones en el área específica. Treinta y seis empresas (20\%) mencionan los circuitos y tours como su actividad más buscada. A continuación, el 17\% tienen las multiactividades como referencia para sus clientes. El senderismo y los deportes acuáticos (piragüismo y buceo) también presentan alguna relevancia en la presente investigación.

\section{Figura 2}

\section{PRODUCTOS/ACTIVIDADES MÁS DEMANDADAS}

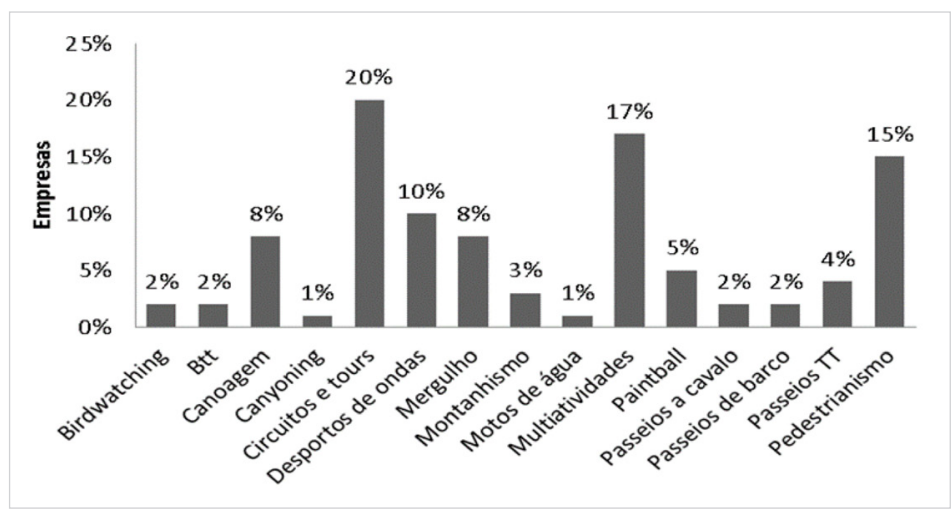

En cuanto a las bases de operación que las empresas disponen, además de su sede social, constatamos que la mayoría $(49,7 \%)$ no tiene ninguna base de operación, 69 (37.7\%) afirman tener una base de operación y sólo 7 (3,8\%), admite tener cuatro bases de operación distintas, además de la sede social.

\subsection{Número de trabajadores}

En el caso de los trabajadores fijos, que noventa empresas $(49,2 \%)$ tienen un trabajador y sesenta y seis empresas $(36,1 \%)$ cuenta en sus cuadros con dos trabajadores fijos. Sólo cuatro empresas $(2,2 \%)$ tienen cuatro trabajadores fijos y sólo una $(0,5 \%)$ tiene cinco trabajadores de ese tipo. 
Tabla 2

TRABAJADORES FIJOS

\begin{tabular}{cccc}
\hline Item & $\mathbf{n}$ & Frec & $\%$ \\
\hline & 0 & 4 & $2,2 \%$ \\
& 1 & 90 & $49,2 \%$ \\
Trabajadores fijos & 2 & 66 & $36,1 \%$ \\
& 3 & 18 & $9,8 \%$ \\
& 4 & 4 & $2,2 \%$ \\
& 5 & 1 & $0,5 \%$ \\
\hline & Total & $\mathbf{1 8 3}$ & $\mathbf{1 0 0 \%}$ \\
\hline
\end{tabular}

En cuanto a los trabajadores discontinuos, la situación es muy parecida, el $42,1 \%$ de la muestra afirma que no tiene ningún colaborador en estas condiciones. Cuarenta y tres de las empresas $(23,5 \%)$ tienen uno y sólo veinte $(4 \%)$ tienen cuatro o más trabajadores discontinuos. En el caso de los trabajadores temporales, el 88,5\% de la muestra tiene entre cero y cinco trabajadores en estas condiciones, mientras que el 59\% tiene dos o menos trabajadores. También se observa que nueve empresas (4,9\%) tienen 10 o más trabajadores temporales (tabla 3).

Tabla 3

TRABAJADORES TEMPORALES

\begin{tabular}{cccc}
\hline Item & $\mathrm{n}$ & Frec & $\%$ \\
\hline Trabajadores temporales & 0 & 41 & $22,4 \%$ \\
& 1 & 31 & $16,9 \%$ \\
& 2 & 36 & $19,7 \%$ \\
& 3 & 10 & $5,5 \%$ \\
& 4 & 15 & $8,2 \%$ \\
& 5 & 29 & $15,8 \%$ \\
& 6 & 3 & $1,6 \%$ \\
& 7 & 4 & $2,2 \%$ \\
& 8 & 3 & $1,6 \%$ \\
\hline
\end{tabular}




\begin{tabular}{cccc}
\hline Item & $\mathrm{n}$ & Frec & $\mathbf{\%}$ \\
\hline Trabajadores temporales & 9 & 2 & $1,1 \%$ \\
& 10 & 3 & $1,6 \%$ \\
& 11 & 2 & $1,1 \%$ \\
& 16 & 3 & $1,6 \%$ \\
& Total & $\mathbf{1 8 3}$ & $\mathbf{1 0 0 \%}$ \\
\hline
\end{tabular}

\subsection{Volumen económico y perfil de intervención de las empresas encuestadas}

En cuanto al volumen económico anual de las empresas participantes en el estudio, se verificó que $30 \%$ de los casos se señala 0 euros, lo que puede significar que las empresas no tuvieron actividad, u optaran por ser evasivas en la respuesta, no indicando el volumen económico. Observando la figura 3, se constató que el 35\% de la muestra tiene una facturación anual de $1 €$ a $15.000 €$ y un $21 \%$ más de $45.000 €$.

Para estos resultados, puede contribuir a la estacionalidad existente en el sector, donde la demanda por este tipo de servicios/actividades, es en su mayoría, resultante de 4 o 5 meses de trabajo por año, especialmente en el período de primavera y verano. De esta forma, nos parece que el volumen de negocios presentado por encima de los $45000 €$, es un poco bajo.

Figura 3

\section{VOLUMEN ECONÓMICO (N=183)}

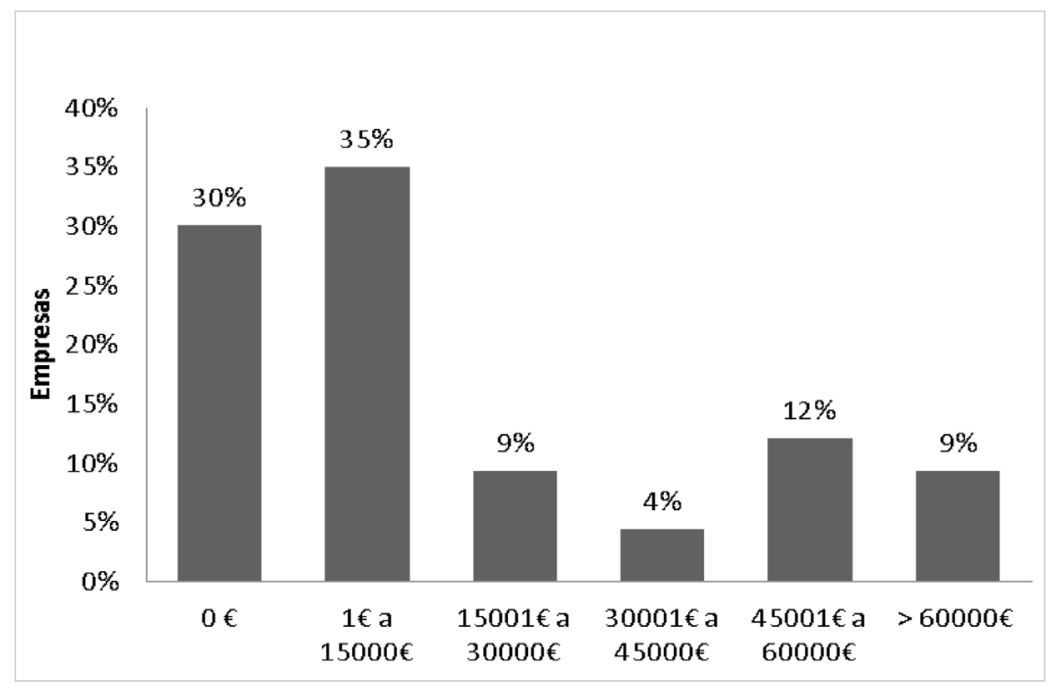


En lo que se refiere al perfil de intervención, la mayoría de las empresas encuestadas comentaron que su intervención, gestión empresarial y actividades que se desarrollaron mayoritariamente dentro del sector del turismo, con 129 (70,5\%) empresas a evidenciar su opción por esa área.

\subsection{Análisis descriptivo de los parámetros multidimensionales y preguntas comple- mentarias (opinión de los empresarios participantes en el estudio)}

En este punto, se procedió al análisis descriptivo de los resultados de los parámetros multidimensionales y preguntas complementarias, de acuerdo con las respuestas dadas por los responsables de las empresas encuestadas. En la tabla 4, el ítem más valorado por las empresas en cada uno de los parámetros multidimensionales, observado a través del porcentaje de respuestas positivas (porcentaje de sî) y la importancia atribuida, expresada a través del valor medio de las respuestas (a través de una escala de likert).

\section{Tabla 4}

ANÁLISIS DE LOS ÍTEMS / PARÁMETROS MÁS VALORADOS

\begin{tabular}{|c|c|c|c|c|c|c|c|}
\hline \multicolumn{8}{|c|}{ Escala de likert } \\
\hline ITEM & & 1 & 2 & 3 & 4 & 5 & $\begin{array}{l}\text { Valor } \\
(\% \text { si) }\end{array}$ \\
\hline \multicolumn{8}{|c|}{ ENTIDAD (2.5.- Poseer una herramienta para medir la satisfacción del cliente.) } \\
\hline & Freq. & 0 & 15 & 18 & 67 & 83 & \\
\hline & $\%$ & $0 \%$ & $8,2 \%$ & $9,8 \%$ & $36,6 \%$ & $45,4 \%$ & $86 \%$ \\
\hline \multicolumn{8}{|c|}{$\begin{array}{c}\text { ATIVIDAD “Estrella” (3.7.- Conocer la normativa específica y actual que regula esta } \\
\text { actividad) }\end{array}$} \\
\hline & Freq. & 0 & 0 & 4 & 51 & 128 & \\
\hline & $\%$ & $0 \%$ & $0 \%$ & $2,2 \%$ & $27,9 \%$ & $69,9 \%$ & $100 \%$ \\
\hline \multicolumn{8}{|c|}{ RECURSOS MATERIALES (4.3.- Sustituir el material por deterioro) } \\
\hline & Freq. & 0 & 0 & 0 & 15 & 168 & \\
\hline & $\%$ & $0 \%$ & $0 \%$ & $0 \%$ & $8,2 \%$ & $91,8 \%$ & $100 \%$ \\
\hline \multicolumn{8}{|c|}{ SEGURIDAD (5.7.- Poseer, los responsables de la actividad, otros elementos de seguridad) } \\
\hline & Freq. & 0 & 0 & 5 & 46 & 132 & \\
\hline & $\%$ & $0 \%$ & $0 \%$ & $2,7 \%$ & $25,1 \%$ & $72,1 \%$ & $99,5 \%$ \\
\hline \multicolumn{8}{|c|}{$\begin{array}{c}\text { MEDIO AMBIENTE NATURAL (6.1.- Conocer la normativa medioambiental en cuanto al } \\
\text { espacio se refiere) }\end{array}$} \\
\hline & Freq. & 0 & 0 & 5 & 38 & 140 & \\
\hline & $\%$ & $0 \%$ & $0 \%$ & $2,7 \%$ & $20,8 \%$ & $76,5 \%$ & $\mathbf{9 8 , 9 \%}$ \\
\hline
\end{tabular}




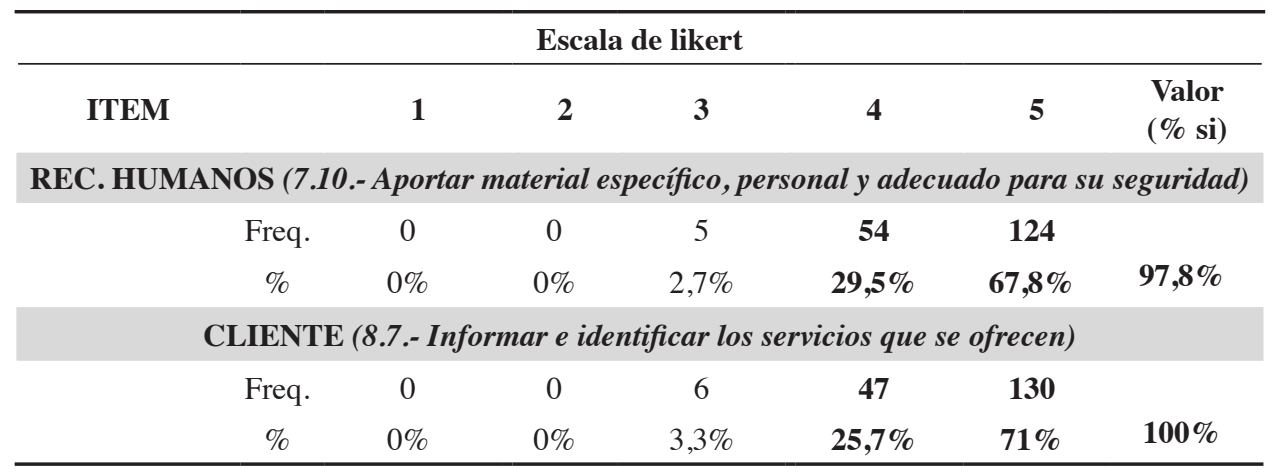

En el análisis a la tabla anterior, la elevada importancia dada por los responsables de las empresas de turismo activo a los ítems 3.7.- Conocer la normativa específica y actual que regula esta actividad (parámetro Actividad "Estrella"); 4.3.- Sustituir el material por deterioro (parámetro Recursos Materiales); 5.7.- Poseer, los responsables de la actividad, otros elementos de seguridad (parámetro Seguridad); 6.1.- Conocer la normativa medioambiental en cuanto al espacio se refiere (parámetro Medio Ambiente Natural) y 8.7.- Informar e identificar los servicios que se ofrecen (parámetro Cliente).

Las respuestas obtenidas en el bloque de preguntas complementarias del cuestionario HEVA, verificamos que el "Atención al cliente" $(4,86)$, la "Profesionalidad" $(4,85)$, la "Satisfacción del producto" $(4,79)$, la "Amabilidad" $(4,75)$ y la "Efectividad en el servicio" $(4,66)$, relativos a la media de las respuestas, fueron los elementos considerados más importantes en este estudio (de acuerdo con el promedio de respuestas dadas) (figura 4).

\section{Figura 4 \\ MEDIA DE RESPUESTAS OBTENIDAS EN EL BLOQUE DE PREGUNTAS COMPLEMENTARIAS}

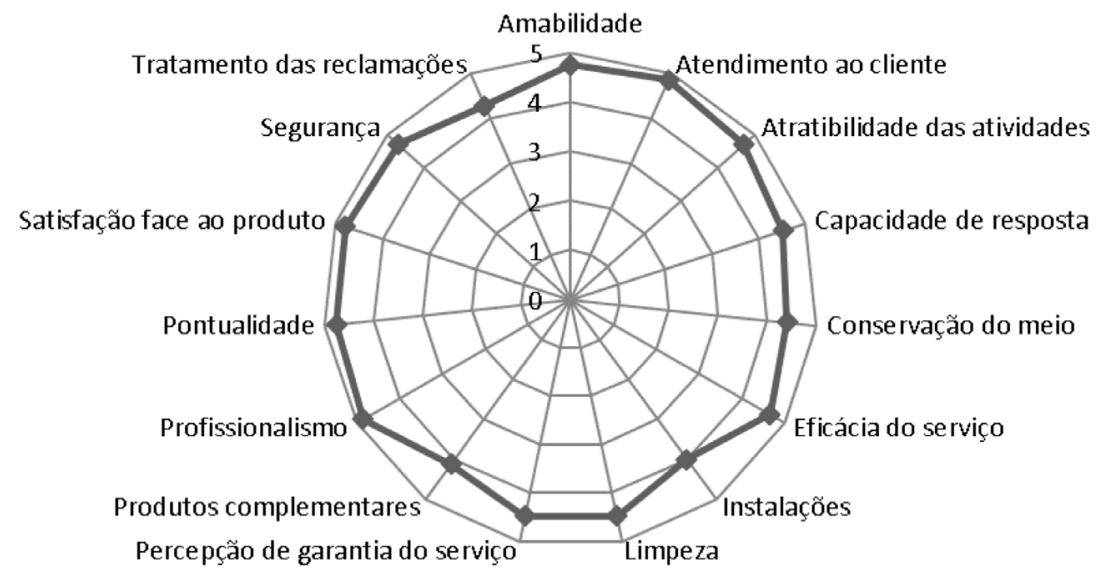


El análisis de los resultados presentados anteriormente, se encontraron que en el parámetro multidimensional "entidad", las empresas de turismo activo encuestadas dan gran importancia al ítem 2.5 - Poseer una herramienta para medir la satisfacción del cliente. Esta posición es asumida por otros autores y está presente en los estudios de Carvalhinho (2006); Cooper (2006); Chelladurai et al. (2000). La satisfacción del cliente también es referenciada por algunos autores cuando señalan la importancia de poseer servicios adaptados a la capacidad de funcionamiento y localización del negocio, infiriendo directamente estos aspectos, en la satisfacción y gestión del cliente (Dabholkar et al., 2000; Buckley, 2010; Sánchez et al., 2011; Cook et al., 2012).

En el parámetro "Actividad Estrella" se referenció el ítem 3.7-Conocer la normativa específica y actual que regula esta actividad, que se configuró como el más importante para los empresarios de turismo activo de la presente investigación. De acuerdo con Miranda (2007), existen varias razones para el abandono del cliente en relación con los servicios que consume, en particular por fallos del servicio o falta de tecnología e innovación. En general, para los empresarios de turismo activo, es importante que exista una actividad/producto diferenciador en sus servicios, que destaca sobre las demás, haciendo que exista siempre que sea posible, alguna innovación o novedad (Grönroos, 1984; Sharifi et al.; Silva, 2013; UNWTO, 2014).

La importancia atribuida a los "Recursos Materiales" los resultados mostraron un acuerdo unánime, como lo demuestra la importancia concedida al ítem 4.3.- Sustituir el material por deterioro, uno de los más valorados por los empresarios portugueses. Estos datos revelan, como ya hemos señalado, la importancia de la gestión diaria de los recursos materiales. Una de las conexiones principales del cliente con la empresa, es realizada través de los materiales, siendo a menudo factor decisivo en cuanto al bienestar y la seguridad de la actividad, y también, del posible retorno o abandono del consumidor (Yan et al., 2013; SPARC'S, 2007; Toplis, 2007).

Los resultados obtenidos en el parámetro "Seguridad", demuestran la importancia de todos los ítems, de acuerdo con la naturaleza de las actividades de turismo activo. Según Marinho et al. (2003), cuanta menos discrepancia haya entre el riesgo percibido y el riesgo real, menos peligros hay con la vivencia de esa práctica. El aumento exponencial de la demanda de este tipo de actividades hace que los responsables de las empresas decidan cada vez más invertir en todos los aspectos relacionados con la seguridad de sus recursos humanos y materiales. La búsqueda por una "Aventura Segura”, referenciada por Melo (2009), lleva a que el riesgo presente en los programas de actividades sea lo más reducido y objetivo posible, sin eliminarlo, pues de esta forma estaríamos desvirtuando la esencia de este tipo de actividades, donde la adrenalina, las emociones y la auto-superación están siempre presentes.

En cuanto al parámetro "Medio Ambiente Natural", los resultados indican que las empresas portuguesas de turismo activo conceden gran importancia al ítem 6.1.- Conocer la normativa medioambiental en cuanto al espacio se refiere, coincidiendo con estudios de Pukkala et al. (1995); McCool et al. (2007); Manning (2011), donde los autores refuerzan la importancia de gestionar y preservar el medio ambiente, en el cual se desarrollan experiencias y actividades de recreación importantes para la región donde se desarrollan. La diversidad de oportunidades que los territorios ofrecen, permite a los responsables de las empresas de turismo activo, construir productos y servicios de calidad (McCool et al., 2007). 
Los parámetros multidimensionales "Recursos Humanos" y "Cliente" son también de gran importancia para los encuestados, con resultados que valoran la importancia dada por los responsables de las empresas a sus empleados, que son la imagen de la empresa, capaces de influenciar de forma positiva o negativa, la percepción y opinión del cliente en relación a los servicios o actividades que realiza (Carvalhinho, 2006). La formación y experiencia de los recursos humanos en cualquier empresa, es uno de los pilares base de sustentación y perdurabilidad de cualquier negocio, de acuerdo con Hui et al. (2007); Shonk y Chelladurai (2008), siendo impulsores y generadores de satisfacción en el cliente (Weiermair; Fuchs, 1999; Yoon; UYSAL, 2005), resultando así en un consecuente retorno y fidelización del mismo.

Siendo el tema central de esta investigación, la calidad, se vuelve fundamental intentar percibir cuál es la postura y feedback, proporcionados por parte del cliente, después de la realización del servicio / actividad, como refieren autores como Tsitskari et al. (2006); Weaver (2006); Tremont y Hernández (2010); Thomas et al. (2011); Sánchez y Currás (2011); Sharifi et al. (2013). Al revés, existe el riesgo directo de interferir en la calidad de todo el servicio/actividad (Buhalis, 2000, Berno, Bricker, 2011).

Por último, observando los datos obtenidos en las respuestas a las preguntas complementarias, que han descrito los empresarios encuestados sobre los factores influyentes de la calidad de los servicios, desde la perspectiva del cliente, destacaron los ítems Atención al cliente, la Profesionalidad y el Tratamiento de las reclamaciones. De acuerdo con la presente investigación y la literatura consultada, estos 3 factores influyeron, de forma positiva o negativa, en la calidad del servicio, por parte del cliente (en opinión de los responsables). Un estudio reciente de Romiti y Sarti (2016), relativo a los factores influyentes en la capacidad de decisión de compra de los consumidores de servicios de Turismo Activo, aborda el encuadramiento técnico y profesional como condición esencial para el retorno del cliente. La capacidad de producir experiencias positivas, donde la relación interpersonal y la capacidad de auto-superación están presentes, hace la gestión del cliente más adecuado al servicio que se produce (Richards, 2011; Romiti, Sarti, 2016).

\section{CONCLUSIONES}

El objetivo de este trabajo fue describir el perfil de las empresas portuguesas de turismo activo y de la percepción de los empresarios, sobre la calidad de los servicios que producen. Con la aplicación del cuestionario HEVA (Bento et al, 2017), destacamos las siguientes conclusiones:

- El reciente crecimiento de este sector en Portugal, donde las empresas están a cargo de uno o dos empresarios y ofrecen hasta 10 actividades / productos al mercado. Desarrollan su actividad durante los doce meses del año.

- Los responsables de las empresas de turismo activo en Portugal participantes en el estudio, consideran muy importante desarrollar herramientas fiables, capaces de medir la satisfacción del cliente - factor importante en la mejora de los servicios prestados por ese tipo de empresas.

- Las empresas portuguesas de turismo activo demuestran una preocupación constante en la comunicación con el cliente, todas las características inherentes a la actividad, en particular con respecto a los aspectos de gestión y minimización de riesgos. 
- Poseer y demostrar los requisitos técnicos para la realización de las actividades, así como conocer y dominar todos los aspectos específicos reguladores (por ejemplo, equipos, seguridad, niveles de dificultad, etc.) de cada una de ellas, es un factor importante para la mayoría de las empresas encuestadas.

- El intercambio de información con los clientes, así como la colocación de demandas concretas para la realización de actividades, son factores esenciales de la seguridad y la calidad, de acuerdo con las empresas portuguesas encuestadas.

- En relación con los aspectos del "Medio Ambiente Natural", la mayoría de las empresas considera poco importante informar las autoridades ambientales en relación a sus actividades cuando éstas se desarrollan en espacios delimitados o regulados (por ejemplo, Parques Naturales).

- El principal factor que determina el éxito de las actividades / servicios prestados en el sector de turismo activo es, según los empresarios portugueses encuestados, la valorización y especialización de sus recursos humanos.

- La gestión y motivación de los recursos humanos son piezas esenciales para la calidad en los servicios prestados. De esta forma, en la gran mayoría de las empresas encuestadas, existe una baja rotación de personal.

- Consideran además que sus clientes dan mucha importancia a la forma en que son conducidos, desde la compra a la realización de la actividad, al profesionalismo y eficacia demostrados en todo el proceso, resultando en una gran satisfacción frente al producto. Según los mismos, si se descuida alguno de los aspectos y actitudes anteriormente mencionados, puede fácilmente resultar en una pérdida de clientes y servicios.

\section{BIBLIOGRAFÍA}

ALVES, A. (2010): Turismo activo: Um produto do turismo e do desporto". (Mestrado), Universidade da Madeira.

ARCOS, A. (2004): "Turismo Rural y Activo a tu alcance". En Y. Lazaro (Ed). Ocio, inclusión y discapacidad. Bilbao, Universidad de Deusto, pp. 329-340.

BENTO, P., SÁEZ-PADILLA, J., FUENTESAL, J. y MURTA, L. (2017): "Versão Portuguesa do Questionário HEVA-Qualidade dos Serviços das Empresas de Turismo Ativo em Portugal". Exedra: Revista Cientíca, nº 1, pp. 115-133.

BEEDIE, P. y HUDSON, S. (2003): "Emergence of mountain-based adventure tourism". Annals of Tourism Research, vol. 30 (3), pp. 625-643.

BELL, S., TYRVÄINEN, L., SIEVÄMEN, T., PRÖBSTL, U. y SIMPSON, M. (2007):

"Outdoor Recreation and Nature Tourism: A European Perspective". Living Reviews in Landscape Research, 1.

BERNO, T. y BRICKER, K. (2011): "Sustainable Tourism Development: The long road from theory to practice". International Journal of Economic Development, vol. 3 (3), pp. 1-18.

BUHALIS, D. (2000): "Marketing the competitive destination of the future". Tourism Management, vol. 21, pp. 97-116.

BUCKLEY, R. (2010): Adventure Tourism Management. Great Britain, Elsevier. 
CARVAlHINHO, L. (2006): Os Técnicos e as Actividades de Desporto de Natureza Análise da formação, funções, e competências profissionais. Portugal, Universidade de Trás-os-Montes e Alto Douro.

CARVALHINHO, L., SEQUERIA, P., SERÔDIO-FERNÁNDEZ, A. y RODRIGUEZ, J. (2011): "A emergência do sector de desporto de natureza e a importância da formação". EFDeportes, 14.

CHELLADURAI, P. y CHANG, K, (2000): “Targets and standards of quality in sport services". Sport Management Review, vol. 3 (1), pp. 1-22.

COOK, M. (2012): "Receptivity to the production of product service systems in the UK construction and manufacturing sectors: a comparative analysis". Journal of Cleaner Production, vol. 32, pp. 61-70.

CUNHA, S. (2006): Actividades Físicas de Aventura na Natureza em Portugal- Passos para a sua compreensão. o caso da cidade de Rio Maior. Porto, Universidade do Porto.

DABHOLKAR, P., SHEPHERD, D. y TORPE, D. (2000): “A comprehensive framework for service quality: investigation of critical conceptual and measurement issues through a longitudinal study". Journal of Retailing, vol. 76 (2), pp. 139-173.

FONSECA, J. (2008): "Os Métodos Quantitativos na Sociologia: Dificuldades de uma Metodologia de Investigação". Paper presented at the VI Congresso Português de Sociologia: Mundos Sociais saberes e Práticas. Lisboa.

GARCIA, M. (2001): Los españoles y el deporte: prácticas y comportamientos en la última década del siglo XX. Madrid, Ministerio de Educación Cultura y Deporte, Consejo Superior de Deportes.

GRÖNROOS, C. (1984): “A service quality model and its marketing implications”. European Journal of Marketing, vol. 18 (4), pp. 36-44.

HEINEMANN, K. (2008): "Introducción a la metodología de la investigación empírica". Barcelona, Paidotribo.

MAROCO, J. (2011): Análise estatística com SPSS Statistics. Portugal, ReportNumber.

MEDIAVILLA, L. (2010): Calidad del servicio del turismo activo (TA) en España (Huesca-Lérida), Italia (Tentino-Alto Adige) y Costa Rica (Alajuela-Cartago): Diseño de un método de valoración de la calidad técnica emitida. Madrid, Universidad Politécnica de Madrid.

MEDIAVILLA, L., GÓMEZ, V., SÁNCHEZ, A. y VILLOTA, S. (2014): "Perfil identificativo de las empresas de turismo de Aventura en España, Italia y Costa Rica". Journal of Sport and Health Research, vol. 6 (2), pp. 177-190.

MELO, R. (2009): Desportos de Natureza: reflexões sobre a sua definição conceptual. Coimbra, FCDEF.

MIRANDA, C. (2007): Qualidade do serviço e satisfação do cliente. O caso Vodafone. Porto, Universidade Católica Portuguesa.

McCOOL, S. y BUSHELL, R. (2007): Tourism and protected areas: benefits beyond boundaries. The Vth IUCN World Parks Congress. Sydney, University of Western Sydney.

ROMITI, A. y SARTI, D. (2016): “Service Quality Experience and Customers Behavioural Intentions in Active Sport Tourism”. Modern Economy, vol. 7 (12), pp. 1.361-1.384. 
RICHARDS, G. (2011): "Creativity and tourism: The State of the Art". Annals of Tourism Research, vol. 38 (4), pp. 1225-1253.

SÁNCHEZ, I. y CURRAS, R. (2011): "Effects of dissatisfaction in tourist services: The role of anger and regret". Tourism Management, vol. 32 (6), pp. 1.397-1.406.

SHARIFI-TEHRANI, M., VERBIC, M y CHUNG, J.Y. (2013): “An analysis of adopting dual pricing for museums: the case of the national museum of Iran". Annals of Tourism Research, vol. 43, pp. 58-80.

SILVA, F. (2013): Turismo na natureza como base do desenvolvimento turístico responsável nos Açores. Portugal, Universidade de Lisboa.

SHONK, D. y CHELLADURAI, P. (2008): “Service Quality, Satisfaction, and Intent to Return in Event Sport Tourism". Journal of Sport Management, vol. 22 (5), pp. $587-$ 602.

SPARC'S (2007): Outdoor Activities. Guidelines for leaders. Ministry of Education, Sports \& Recreation, New Zealand.

UNWTO (2014): Global Report on Adventure Tourism. World Tourism Organization Reports.

WEIERMAIR, K. y FUCHS, M. (1999): "Measuring tourist judgment on service quality". Annals of Tourism Research, vol. 26 (4), pp. 1.004-1.021.

WEAVER, D. (2006): Sustainable tourism: Theory and practice. Oxford, Elsevier.

YAN, L. y MCKERCHER, B. (2013): "Travel culture in eastern Jin China (317-420 $\mathrm{ad)}$ : the emergence of a travel culture of landscape appreciation". Annals of Tourism Research, vo. 43, pp. 20-36.

YOON, Y. y UYSAL, M. (2005): "An examination of the effects of motivation and satisfaction on destination loyalty: A structural model". Tourism Management, vol. 26 (1), pp. 45-56. 
